\title{
LA SOCIEDAD DE LA INFORMACIÓN Y LA FORMACIÓN DEL PROFESORADO. E-ACTIVIDADES Y APRENDIZAJE COLABORATIVO
}

\author{
(INFORMATION SOCIETY AND TEACHER TRAINING. E-ACTIVITIES AND COLLABORATIVE \\ LEARNING)
}

\author{
Miguel A. Martín Sánchez \\ Universidad de Extremadura (España) \\ Eloy López Meneses \\ Universidad Pablo de Olavide (España)
}

\section{RESUMEN}

Los espacios virtuales educativos se muestran como nuevas comunidades y contextos de aprendizaje en los que es posible la interacción y relación educativa sin apenas límites espaciales, geográficos y temporales. Internet se perfila como una herramienta universal para el profesorado de todos los niveles en la búsqueda, el intercambio de información, las experiencias formativas y la investigación. Dentro de este nuevo espacio educativo, las actividades más adecuadas son aquellas que invitan a la construcción del conocimiento, la experimentación y la resolución de problemas, a escala individual y grupal. También, aquéllas que vayan dirigidas a relacionar los conocimientos previos de los estudiantes con los nuevos contenidos del curso virtual, las que favorezcan el aprendizaje autónomo junto a las que inviten a buscar diferentes alternativas sobre una problemática. En este artículo reflexionaremos sobre las principales actividades didácticas utilizadas en los espacios virtuales de educación desde el punto de vista del aprendizaje colaborativo.

Palabras clave: educación, internet, actividades, aprendizaje colaborativo.

\begin{abstract}
In this paper, virtual education areas are depicted as new learning communities and contexts, i.e. where (human) interaction and the forging of educational relationships are possible. This does not include, however, other spatial, geographical or temporal limitations. In this study, the Internet is presented as a universal tool for both teaching staff and general users. It is used for searching for specific information, exchanging ideas, or discussing research. Within the context of this new virtual educational area, the most suitable activities are those that foster the creation of knowledge and that promote experimentation and problem solving in an individual or group context. It also refers to activities that
\end{abstract}


combine students' previous knowledge with the new contents of virtual courses. In this way, there is greater autonomous learning and problem solving of specific theoretical questions. We also examine core didactic activities that are used in virtual education areas, that is, from the view of collaborative learning.

Keywords: education, the internet, activities, collaborative learning.

Como punto de partida inicial consideramos con Travé, Pozuelos y Cañal (2006) que la enseñanza transmisiva tradicional, aún hoy mayoritaria en el panorama educativo de nuestro país, constituye una herencia cultural de los siglos XIX y XX, y resulta, sin duda, un modelo educativo que no puede satisfacer los requerimientos de la sociedad actual.

Desde la perspectiva integradora, los procesos formativos son más interactivos y constructivos, donde el estudiante es el protagonista de su proceso de aprendizaje y brilla con luz propia los procesos formativos donde se trabajan con las ideas y experiencias de los estudiantes en colaboración.

El mundo se encuentra mediatizado, y la época actual se caracteriza por la utilización casi exclusiva de los medios terciarios, es decir, de aquellos que necesitan aparatos técnicos para la emisión, distribución y recepción de la información. El mundo de la información pertenece a los medios de comunicación, quienes a su vez se encuentran tecnificados. Vivimos por lo tanto en una sociedad mediática, tecnificada, en la que los propietarios, productores y gestores de la técnica deciden las posibilidades, amplitud y desarrollo de la comunicación. Si la educación es información y comunicación, resulta evidente la relación: la educación se encuentra, a día de hoy en nuestra sociedad, mediatizada por los medios de comunicación.

\section{FORMACIÓN DEL PROFESORADO Y ESPACIOS VIRTUALES DE EDUCACIÓN}

La formación del profesor va unida al desarrollo profesional del docente. No es una cuestión nueva. Se trata de concebir la formación como un aprendizaje constante, de modo que la formación se acerque al desarrollo de actividades profesionales y a la práctica profesional y desde ella (Imbernón, 2004). Se considera por tanto la práctica de la enseñanza como una profesión dinámica, en continuo desarrollo, por lo que la formación y el reciclaje son aspectos muy importantes que todos los profesores deben asumir como propios. 
Ahora bien, esta formación y reflexión didáctica del profesor debe estar sustentada en principios científicos, y la praxis didáctica debe desarrollarse a partir de la formación continua y la experiencia cotidiana, así como las reuniones y el trabajo en equipo con otros profesionales del mundo de la educación (Aebli, 1988).

En cuanto a los tiempos que corren, la formación y desarrollo profesional del docente, y tal y como expusimos en el Congreso Internacional Edutec 2004, ha cambiado. Ya no basta con saber, sino que también es necesario un saber vinculado con los profundos cambios económicos y sociales en marcha, con las nuevas tecnologías, con la nueva organización industrial e institucional, en un mundo crecientemente complejo e interdependiente, que requiere personas con viva inquietud creativa e innovadora, con espíritu crítico, reflexivo y participativo (Ballesteros, López Meneses y Torres, 2004).

Los futuros profesores demandan cada vez más la necesidad de incorporar las nuevas tecnologías y los medios de comunicación en los planes curriculares (Sevillano García, 1997), lo cual evidencia la importancia y necesidad de una formación nueva e innovadora.

La educación, como parte de la Sociedad, acusa y responde a los cambios y novedades, retos y desafíos que le propone los tiempos modernos. Es un hecho evidente que Internet ofrece grandes posibilidades de comunicación e interacción, y que tal y como señala García Aretio (2006), la relación entre sociedad y tecnología está variando las relaciones de los seres humanos en el seno comunitario.

Los espacios virtuales educativos, en definitiva, se muestran como nuevas comunidades y contextos de aprendizaje en los que es posible la interacción y relación educativa sin apenas límites espaciales, geográficos y temporales. En palabras de Sánchez Bravo (2001) la educación ha cambiado, ya no se limita a un espacio temporal concreto, sino que se prolonga a lo largo de la vida, ofreciendo las TIC importantes ventajas, en un mundo en el que el reciclaje y la adquisición de nuevos conocimientos devendrá fundamentalmente "por una mera cuestión de supervivencia en el nuevo medio ambiente tecnológico, caracterizado por la rapidez en la circulación y mutación de las informaciones" ${ }^{\prime}$.

Coincidimos con la experta en tecnología educativa Luisa Torres (2005), que la sociedad ha cambiado considerablemente en un corto periodo de tiempo y continua haciéndolo apresuradamente. Éstos cambios no sólo se apoyan en un aumento cuantitativo de la información disponible por el avance de las "nuevas" tecnologías 
y en la inmediatez con que dicha información puede llegar a los destinatarios, sino que estimamos que están directamente relacionados con todas las modificaciones culturales, es decir, cambios en la forma de comunicarnos, de trabajar, de divertirnos, de aprender, etc.

Como expusimos recientemente, en una publicación internacional, Cabero, Ballesteros y López Meneses (2004), Internet se va convirtiendo, inexorablemente, en una especie de tejido nervioso que se va desarrollando rápidamente en nuestras sociedades y se perfila como una herramienta universal para el profesorado de todos los niveles en la búsqueda, el intercambio de información, las experiencias formativas y la investigación.

Resulta evidente que en la actualidad la escuela ha perdido el monopolio de la educación. En los tiempos que corren, los procesos educativos no son privativos de la escuela como organización, y otras gentes y canales han ampliado, multiplicado y ensanchado los límites tradicionales que imperaban en la estructura formal de la escuela. La educación tiene lugar, a día de hoy y para la mayoría de los individuos, en variados lugares y espacios, a través de múltiples canales, con el empleo de códigos diversos, con técnicas y metodologías heterogéneas, y con gran variedad de soportes que superan los confines de la escuela. No es que la escuela haya dejado de ser útil o no cumpla con su papel asignado, sino que, como afirma Antonio Bartolomé (1996), la "escuela, la enseñanza secundaria e incluso la universidad no están preparadas para afrontar el gran reto del final del milenio: el paso de la sociedad industrial a la sociedad de la comunicación está suponiendo un nuevo modo de conocer"2, afirmación que en cualquier caso habría que matizar. Y es preciso matizar porque en modo alguno son términos dicotómicos o enfrentados escuela-sociedad de la información, sino que habrá que buscar el equilibrio y el nuevo lugar que ésta le deja a aquella.

Teniendo en cuenta todo lo anterior, podemos sacar algunas conclusiones relevantes para la educación en relación con el mundo de la información y la sociedad de la información.

La primera reflexión que debemos hacer parte del planteamiento sistémico de la educación, como subsistema del sistema sociocultural. La consideración de la sociedad como un sistema en el que los individuos se interrelacionan entre sí mediante los organismos creados para ellos, con unos objetivos comunes, hace preciso analizar el papel de la educación dentro de ese sistema denominado sociedad. Por lo tanto, la educación emerge como un subsistema de la sociedad, 
un sistema abierto que, insertado en un medio humano y sociocultural complejo y pluriforme, se relaciona e interactúa abiertamente con otros subsistemas y, por ende, con la sociedad entera. Apuntan García Carrasco y García del Dujo (1996) que cuando hablamos de educación, y de hechos educativos, hay que partir de un punto de vista inicial insoslayable, que es aquel que hace referencia a que los hechos educativos son hechos y acontecimientos del mundo, y por lo tanto la relación con otros sistemas abiertos, con lo que intercambia elementos y recibe influencias en sus procesos, motivo por el cual es preciso tener en cuenta las diferentes relaciones entre la educación y la sociedad.

La sociedad influye en la educación, y ésta en la sociedad. A través de la comunicación y de las relaciones entre los individuos de una misma comunidad, el hombre llega a poseer las cosas que necesita para su supervivencia, y la dimensión individual del hombre, su aparente incomunicabilidad, se comparte en una relación con los demás. Mediante la comunicación, el ser humano (incomunicable en una dimensión profunda) compartesuincomunicabilidad con los demás, transformándose en un ser social, y la sociedad consigue que el carácter individual del hombre permanezca, pero que trascienda hacia una relación mayor y más compleja.

La segunda reflexión hace referencia al papel de los medios de comunicación en la sociedad actual. El mundo se encuentra mediatizado, y la época actual se caracteriza por la utilización casi exclusiva de los medios terciarios, es decir, de aquellos que necesitan aparatos técnicos para la emisión, distribución y recepción de la información. El mundo de la información pertenece a los medios de comunicación, quienes a su vez se encuentran tecnificados. Vivimos por lo tanto en una sociedad mediática, tecnificada, en la que los propietarios, productores y gestores de la técnica deciden las posibilidades, amplitud y desarrollo de la comunicación. Si la educación es información y comunicación, resulta evidente la relación: la educación se encuentra, a día de hoy en nuestra sociedad, mediatizada por los medios de comunicación. Una última reflexión más hace referencia al papel que juegan las nuevas tecnologías en el procedo de adquisición de conocimientos, en el aprendizaje y la educación. Educar es comunicar, y es que la relación entre los medios de comunicación y la educación es muy vieja.

La tercera vía de análisis nos lleva a reflexionar sobre las redes de comunicación. Si las culturas y sociedades se basan en la comunicación, es preciso que anotar que la comunicación predominante actualmente se basa en la televisión o en Internet, herramientas que dependen de la técnica, obligando a la tecnificación y reduciendo los medios primarios de la comunicación humana a un segundo papel. 
En los últimos años se está hablando mucho del llamado "cambio cultural”, y resulta evidente que la tecnología está ayudando a ello, al modificar a escala mundial la comunicación humana, los espacios de intercambio y, por supuesto, la validez de la cultura. Son muchos los autores que han alertado sobre esta "amenaza", entre ellos Vincent Mosco (1989), que denuncia el cambio cultural que las nuevas tecnologías provocan en nuestras vidas, ya que los nuevos desarrollos de la comunicación y de la información en relación con las nuevas tecnologías aumentan, en lugar de disminuir, las disparidades sociales, tales como el alfabetismo verbal, visual y de información. Para Mosco el problema ya no es proteger la privacía individual, sino que se presenta como una auténtica amenaza a la propia libertad, a la autodeterminación.

Otra unidad de análisis hace referencia a la intensidad creciente que en los últimos años ha cosechado la ciencia y la tecnología, y como consecuencia de este nuevo cambio tecnológico, la información y la comunicación han reclamado su papel preponderante en la sociedad, viviendo una era de la información o una sociedad de la información, término que bien merece que le dediquemos una líneas por su implicación y consecuencias para la educación.

La sociedad de la información es teletrabajo, teledocumentación, redes, autopistas de la información, nuevas formas de trabajar, televisión digital..., y por supuesto, Internet. Es, en suma, una nueva sociedad, diferente, una sociedad donde los bits y los números mandan y los ordenadores ejecutan. Se trata por lo tanto de un cambio ya presente, al que los profesionales de la educación deben estar atentos y aprovechar las nuevas tecnologías, porque igual que la sociedad industrial cambió a los hombres de aquel tiempo, la sociedad que ahora vivimos modificará la relación de todos los individuos del planeta, la comunicación, el acceso a la información, el trabajo, y por supuesto, la educación: sus planteamientos, contextos, entornos, acciones y procesos.

Un análisis y reflexión más profundo, nos llevaría a analizar, desde una perspectiva pedagógica, la sociedad de la información como una noción ecléctica que recoge diversas teorías sociológicas, caracterizada, desde un punto de vista educativo y cultural, como una sociedad desmasificada e individualizada dentro de una sociedad más amplia.

En definitiva, y retomando todo lo dicho hasta el momento, la actual sociedad de la información ha provocado cambios importantes en el concepto de educación, planteando a los profesionales nuevas estrategias desde, a través y con la información, 
reestructurando sus modos de educar y enseñar, mediatizados y delimitados por este nuevo concepto de sociedad.

Pero sin duda, el fenómeno que más se identifica y que va irremediablemente unido a la sociedad de la información y a las redes de comunicación, es Internet. Tapio Varis (1996) indica como una de las claves del periodo al "liberalización de las telecomunicaciones"yla "explotación dela red Internet por empresas einstituciones"3. Internet es cada vez más accesible, ofrece más utilidades y herramientas, su utilidad es evidente en el trabajo, en las relaciones sociales, en la información y, por supuesto, en las relaciones educativas.

El software social para la formación del profesorado universitario se ha convertido en factor clave en las experiencias universitarias de innovación pedagógica, en el contexto de los nuevos retos del Espacio Europeo (Aguaded y López Meneses, 2009).

En el contexto europeo, no cabe duda que las TIC y especialmente las metodologías en red orientadas bajo el enfoque socio-constructivista e investigador, jugarán un papel muy significativo, por las posibilidades que pueden ofrecer: establecer comunicaciones sincrónicas y asincrónicas entre los diferentes miembros de la comunidad europea, potenciar la construcción de agrupaciones colectivas internacionales de conocimientos, ofrecer experiencias contextualizadas y significativas para el alumno, favorecer la toma de decisiones y la resolución de problemas sociales por los estudiantes, potenciar proyectos de investigación europeos en equipo, etc. (Cabero, Valverde y López Meneses, 2009).

Actualmente las instituciones de Educación Superior, en general, y las españolas en particular, se encuentran en uno de esos periodos que podríamos definir como de encrucijada histórica. Nos atreveríamos a decir que las universidades están inmersas, de modo similar a como ocurre en otras muchas instancias sociales, en un proceso de reconversión o adaptación a las nuevas demandas sociales.

Dentro del marco del Espacio Europeo de Educación Superior (EEES) se manifiesta la necesidad de promover la convergencia entre los diferentes sistemas de educación superior para facilitar a los titulados la integración en un mercado laboral sin fronteras y ofrecer un marco más atractivo para los estudiantes del resto del mundo. Son varios los documentos que orientan esta convergencia europea. 
Sobre este particular existe abundante documentación, pero destacan, a nuestro entender, la declaración de la UNESCO sobre la Educación Superior en el siglo XXI (1998), Declaración de Bolonia (1999), Comunicado de Praga (2001), Comunicado de Berlín (2003), Bergen (2005), Londres (2007). El objetivo que se proponen es conseguir, antes del 2010, el desarrollo progresivo del EEES teniendo como eje, el aprendizaje y el respeto a la diversidad de culturas, lenguas y sistemas educativos y la autonomía universitaria (Montserrat, Gisbert y Sofía, 2007). Con ello se plantea un nuevo contexto universitario en la que se tiende a una creciente movilidad de los estudiantes y de las políticas de participación. El intercambio, la transparencia y la comunicación se convierten en elementos claves para la calidad en las universidades.

Aumenta la heterogeneidad de los alumnos. Tradicionalmente, la universidad se dirigía a una audiencia muy específica. En la actualidad nos encontramos con perfiles de alumnado muy heterogéneo, con características personales, intereses, expectativas y necesidades diferentes.

Se propone un cambio en el paradigma educativo, donde el alumno se sitúa en el centro del proceso del aprendizaje y se manifiesta la necesidad de una formación continua y metodologías orientadas al aprendizaje activo.

Se manifiesta la necesidad de que las universidades, las demás instituciones superiores y sobre todo los estudiantes participen para crear de manera constructiva el Espacio Europeo de Enseñanza Superior.

El perfil académico profesional es definido a través de competencias. Con ello se desprende que la universidad debe dar respuesta a los nuevos requerimientos profesionales (saber, saber hacer, saber estar y ser). Se requiere sistemas de orientación, seguimiento y evaluación como elementos fundamentales para garantizar la calidad.

En este sentido, la formación no sólo se plantea como un requisito para los niños, adolescentes y jóvenes, de modo que se preparen para el futuro, sino también como una necesidad permanente para cualquier individuo al margen de su edad. Asimismo, en los referidos documentos se cuestionan los modelos y estrategias transmisivas de enseñanza basadas en la transmisión del conocimiento por parte del profesor, el aprendizaje memorístico por parte del alumnado y su control a través de exámenes. Por el contrario, se insiste en que los métodos de enseñanza deben potenciar la capacidad de aprendizaje autónomo por parte del estudiante, 
el desarrollo de competencias intelectuales y sociales variadas, las capacidades de resolución de problemas más que la mera reproducción del conocimiento, y el fomento del trabajo colaborativo.

En la Sociedad de la Información y del Conocimiento el desarrollo de la red, Internet, parece traer consigo, junto a otros cambios de distinta naturaleza, la posibilidad de una profunda transformación en el ámbito de la comunicación interpersonal.

\section{E-ACTIVIDADES Y APRENDIZAJE COLABORATIVO}

"El usar TIC para favorecer el aprendizaje colaborativo va más allá de poner a disposición en forma electrónica material para los alumnos y proveer de espacios para la interacción entre ellos y el tutor. Su potencial y real aplicación se da cuando hay actividades pedagógicamente diseñadas en las cuales los estudiantes interactúan para construir conocimiento en forma social” (Barberá, 2001).

Consideramos que las actividades más adecuadas son aquellas que invitan a la construcción del conocimiento, la experimentación y la resolución de problemas, a escala individual y grupal. También, aquéllas que vayan dirigidas a relacionar los conocimientos previos de los estudiantes con los nuevos contenidos del curso virtual, las que favorezcan el aprendizaje autónomo junto a las que inviten a buscar diferentes alternativas sobre una problemática. Igualmente, las que estimulen la reflexión y el análisis de lo aprendido, la toma de decisiones, la negociación de significados, la utilización de herramientas de comunicación y todas las relacionadas con la creación de conocimientos colectivos. Es decir, aquéllas que faciliten el aprendizaje colaborativo.

Begoña Gross y otros autores (2009) en un artículo de muy reciente publicación, afirmaban, con mucho acierto, que los métodos tradicionales de transmisión del conocimiento resultan obsoletos e incompletos, indicando la importancia de las actividades y herramientas que permitan la comunicación y la interacción, apuntando la mejora en el aprendizaje mediante técnicas que faciliten la colaboración ${ }^{4}$.

Coincidimos con Cabero (2003), que colaborar no consiste solamente en la aportación de información o esfuerzo para alcanzar un producto, sino compartir visiones y objetivos, es decir, construir de forma conjunta. En la misma línea de pensamiento, Román (2003), señala que en el momento actual de la educación, el trabajo de grupo realizado de manera colaborativa debe ser un ingrediente esencial 
en las actividades de enseñanza-aprendizaje. La realización de un verdadero aprendizaje colaborativo, no sólo se requiere trabajar juntos, sino cooperar en el logro de una meta que no se puede lograr individualmente.

Para Gros (2004), aprender en colaboración implica un proceso de constante interacción en la resolución de problemas, elaboración de proyectos o en discusiones acerca de un tema en concreto; donde cada participante tiene definido su rol de colaborador en el logro de aprendizajes compartidos y donde el profesor participa como otro colaborador más, pero con ciertas funciones de orientador y mediador, garantizando la efectividad de la actividad colaborativa.

Guitert y Giménez (2000) entienden el aprendizaje colaborativo virtual como un proceso social de construcción de conocimiento, en el que, a partir del trabajo conjunto y el establecimiento de metas comunes, se da una reciprocidad entre un conjunto de individuos que saben diferenciar y contrastar sus puntos de vista de tal manera que llegan a generar un proceso de construcción de conocimiento. Es un proceso en el que cada individuo aprende más de lo que aprendería por sí solo, fruto de la interacción de los integrantes del equipo. En el desarrollo de un grupo, por tanto, la interacción se convierte en un elemento clave, teniendo en cuenta que es el proceso esencial de juntar las contribuciones de los participantes en la creación de conocimiento.

Aprender en colaboración supone entonces que a través de una serie de interacciones se promueve un cambio en la actividad cognitiva del estudiante, permitiendo confrontar sus ideas con las ideas de otros, ya sea por la generación de un conflicto o por la simple descripción de esas ideas.

Comparando los resultados de esta forma de trabajo con modelos de aprendizaje transmisivos, se ha encontrado que los estudiantes aprenden más cuando utilizan el aprendizaje colaborativo, recuerdan por más tiempo el contenido, desarrollan habilidades de razonamiento superior y de pensamiento crítico y se sienten más confiados y aceptados por ellos mismos y por los demás.

En resumen, en los procesos colaborativos los estudiantes tienen unas finalidades en común y, en vez de competir, deben ayudarse y compartir a través de la negociación y el intercambio mutuo, aceptando y debatiendo ideas de una forma colectiva. En este sentido, Cabero (2006) indica que en cierta medida podemos decir que el aprendizaje colaborativo prepara al estudiante para asumir y cumplir compromisos grupales, ayudar a los compañeros, solicitar ayudas a los demás, aprender a aceptar 
los puntos de vista de los compañeros, descubrir soluciones que beneficien a todos, ver puntos de vista culturales diferentes, aprender a aceptar críticas de los demás, exponer sus ideas y planteamientos en forma razonada y familiarizarse con procesos democráticos.

De acuerdo con Cabero y Gisbert (2002) el tipo de actividades propuestas es uno de los elementos más significativos para determinar la calidad de los materiales destinados a la formación.

Bajo nuestra óptica didáctica constructivista e investigadora, en concordancia con las propuestas efectuadas por Cañal (2000) las posibles actividades, ya sean presenciales o virtuales, que pueden corresponder a esta perspectiva son aquellas que cumplan algunos de los siguientes criterios:

- Actividades que fomenten, en general, un papel activo del estudiante.

- Actividades que ayuden al estudiante a elaborar su propio conocimiento a partir de la interacción con otras personas y recursos digitales.

- Actividades que promuevan la formulación de interrogantes susceptibles de someterse a investigación.

- Actividades que inviten a expresar, organizar y contrastar los conocimientos e hipótesis iniciales de los estudiantes sobre los objetos de estudio a investigar.

- Actividades que estimulen el aprendizaje autónomo.

- Actividades relacionadas con la elaboración de proyectos de investigación para dar respuesta a problemas.

- Actividades de exploración de nuevos contenidos por medio de recursos digitales y otras fuentes de información.

- Actividades orientadas a estructurar la información obtenida. Tales como: resumir, comprender, relacionar, concluir, etc.

- Actividades dirigidas a comunicar, debatir o colaborar con otros participantes del curso virtual $\mathrm{u}$ otras personas las tareas y los procesos de aprendizaje desarrollados y obtenidos.

- Actividades que fomenten la aplicación o transferencia de procesos cognitivos/ procedimentales en nuevos escenarios y contextos.

- Actividades encaminadas a la reflexión metacognitiva sobre el desarrollo y los resultados de las investigaciones realizadas. 
Referente a las posibles e-actividades que podemos realizar en un entorno web integrador, destacamos la elaboración de mapas conceptuales interactivos.

Según Novak y Gowin (1999), los mapas conceptuales tienen por objeto representar relaciones significativas entre conceptos en forma de proposiciones. Una proposición consta de dos o más términos conceptuales unidos por palabras para formar una unidad semántica. En su forma más simple, un mapa conceptual constaría tan sólo de dos conceptos unidos por una palabra de enlace para formar una proposición.

Según la enciclopedia de conocimiento abierto, Wikipedia ${ }^{5}$ define el mapa conceptual como una técnica usada para la representación gráfica del conocimiento. Un mapa conceptual es una red de conceptos. En la red, los nodos representan los conceptos, y los enlaces las relaciones entre los conceptos en forma de flechas etiquetadas.

Del Castillo y Barberán (2001) indican que es una herramienta de asociación, interrelación, discriminación, descripción y ejemplificación de contenidos, con un alto poder de visualización. Según Novak (2000), los principales elementos que componen un mapa conceptual son los siguientes:

- Concepto. Se entiende por concepto la palabra o término que manifiesta una regularidad en los hechos, acontecimientos ideas y/o cualidades.

- Proposición. Se establece a partir de la unión de dos o más conceptos ligados por palabras de enlace en una unidad semántica. Corresponde a la unidad principal del significado.

- Palabras de enlace. Son palabras que unen los conceptos y señalan los tipos de relación existente entre ellos.

Finalmente, la representación de los mapas conceptuales está dada por un esquema gráfico en donde se visualizan:

- Los conceptos colocados dentro de una elipse.

- Las palabras enlace que se escribe sobre o junto a una línea que une los conceptos.

En este sentido, Valverde (2002), indica que los mapas conceptuales son herramientas de representación del conocimiento. Sirven para expresar de modo 
gráfico las relaciones significativas entre determinados conceptos que, al combinarse, forma proposiciones.

Estrada y Febles (2000) señalan que los mapas conceptuales resultan muy útiles en las diversas etapas del proceso educativo:

- En la planificación, como recurso para organizar y visualizar el plan de trabajo, evidenciar las relaciones entre los contenidos y resumir esquemáticamente el programa de un curso.

- En el desarrollo, como una herramienta que ayuda a los estudiantes a captar el significado de los materiales que pretenden aprender.

- En la evaluación, como recurso para la evaluación formativa. Permite "visualizar el pensamiento del alumno", para así, corregir a tiempo posibles errores en la relación de los conceptos principales.

Otra actividad que nos interesa resaltar en los entornos de aprendizaje constructivistas e investigadores es la participación por parte de los estudiantes en debates y foros de discusión moderados por el profesor tutor. En ellos consideramos que debe primar la discusión, el diálogo abierto de carácter formal y el intercambio de ideas y experiencias de los estudiantes. El profesor será el moderador del debate, interviniendo enéllo menos posible, guiando el discurso, dinamizandola participación de todos en las propuestas formuladas por sus compañeros y colaborando en la construcción de las conclusiones finales.

El debate puede organizarse a partir de una experiencia o documentación previa y en torno a una cuestión que presente diferentes partes o puntos de vista a tratar (Cabero y Pérez, 2005).

Dichas actividades digitales fomentan el intercambio de opiniones y el análisis, en la mayoría de las veces profundo del objeto de estudio propuesto para el debate en línea. También, posibilita la comunicación entre los profesores y los estudiantes y la de estos entre sí, difundir las propias ideas y sus experiencias cotidianas, valorar las aportaciones de su compañero y contrastar diferentes puntos de vista, hechos y teorías opuestas, desarrollar el pensamiento crítico, adquirir nuevas competencias tecnológicas con su utilización. Además, permite a los alumnos la defensa de sus propias ideas, la crítica de contraposiciones, la negociación social y la construcción de comunidades de conocimientos y experiencias compartidas. 
Referente a la siguiente e-actividad podemos indicar que una simulación educativa puede ser definida como el aprendizaje, a través de la interacción, de un modelo basado en algún fenómeno o actividad. No sólo reproduce un fenómeno, también lo simplifica por omisión, cambio o incorporación de características. Tiene como finalidad favorecer el aprendizaje por descubrimiento y desarrollar las habilidades implicadas en la investigación de un fenómeno de naturaleza física o social. Proporcionan un entorno de aprendizaje abierto y altamente interactivo, basado en modelos reales. Con estos programas el usuario tiene posibilidades para la experimentación y el contraste de variables. Posee la ventaja de no perder realismo, aunque actúe de modo virtual, con lo que ello supone de economía de esfuerzo, tiempo, riesgos y dinero para el proceso de enseñanza. La mejor simulación educativa no tiene porque ser aquella que posea una representación más exacta de la realidad, ya que gracias al uso de modelos simplificados los alumnos pueden aprender a resolver problemas, ejecutar procedimientos, llegar a comprender las características esenciales de un fenómeno y cómo controlarlo, o aprender cómo actuar en determinadas situaciones. En cada caso, el propósito es ayudar a los alumnos a construir sus propios modelos mentales de los fenómenos y los procedimientos, así como proporcionarles oportunidades para explorar, practicar, evaluar y mejorar los modelos de un modo seguro y eficiente. Esto se consigue cuando los modelos están simplificados. Además, las simulaciones educativas pueden añadir elementos que no están presentes en el mundo real.

Por otra parte, una simulación es adecuada para favorecer la comprensión de ideas y conceptos abstractos, así como para el aprendizaje de estrategias o la adquisición de una visión global de una realidad. Una buena simulación es capaz de generar pensamientos y sentimientos profundos en sus participantes.

Siguiendo al mismo autor, las simulaciones son idóneas para manipular y modificar, según las necesidades educativas del momento, las variables espaciotemporales. En una simulación se puede jugar con el tiempo y el espacio. Las simulaciones nos dan la oportunidad de acelerar los efectos de una decisión que podría necesitar un período de tiempo muy dilatado y, por consiguiente, los estudiantes pueden conocer el impacto potencial de determinadas acciones presentes en un futuro (o pasado) más o menos lejano. Por último, las simulaciones son útiles para situarnos en un lugar o en un tiempo donde sería imposible tener una experiencia directa. Son muy buenas para comprender procesos que están fuera de nuestro entorno vital. Las simulaciones son experiencias de inmersión, que encajan mejor para aquellos contenidos que necesitan ser aprendidos en contexto y que requieren la participación activa en la resolución de problemas. 
En definitiva, la implementación de simulaciones en el ámbito telemático ofrece una variedad de posibilidades didácticas muy interesantes desde representar procesos complejos, ensayar procedimientos para la resolución de problemas, potenciar la creatividad y el pensamiento divergente, mostrar las posibles interacciones de fenómenos y experimentos socio-naturales, ampliar el campo de experiencias de los estudiantes, realizar juegos de rol interactivos en escenarios educativos, comprender y analizar procesos educativos difíciles de observar en la realidad hasta ayudar a tomar decisiones. En resumen las simulaciones educativas son interesantes eactividades que permiten el aprendizaje significativo.

Referente a los juegos de rol, por lo general, el profesor-tutor presenta una situación problemática al grupo, en la que intervienen diferentes personajes generalmente opuestos. Cada miembro del grupo escoge un personaje y actúa de forma anónima tal como lo haría el personaje en la realidad. El resultado del juego puede ser una presentación colectiva, un informe o el mismo proceso de discusión. Estas e-actividades permiten al discente aplicar sus conocimientos teóricos a una situación real, simulada, a la vez que se promueve la comprensión de la posición y actitudes de las personas ante ciertos conflictos.

Y, la última actividad que resaltamos como ejemplo de e-actividades que se pueden llevar en entornos telemáticos integradores son aquellas que se orientan a la utilización y/o elaboración de herramientas relacionadas con el software social o con la actual web 2.0.

El concepto Web 2.0 debe su origen a unas ideas a mediados de 2004 entre los equipos de O'Really Media y MediaLive International, dos empresas dedicadas a la producción de eventos, publicaciones y conocimientos sobre las tecnologías digitales $^{6}$.

La Web tradicional se basa en un espacio de publicación corporativo y de servicios. Las comunidades de usuarios se formaban porque se ofrecían servicios, pero existían pocos espacios para que los miembros publicaran sus contenidos. Sólo se podía consumir. La Web 2.0 propone la participación abierta y gratuita basada en un software en línea, permitiendo que el usuario pueda también convertirse en generador de información.

En una sociedad de cambios constantes consideramos que los estudiantes del actual milenio deben relacionarse con las nuevas herramientas digitales que le ha tocado vivir. El estudiante de e-learning deberá dominar una serie de destrezas: 
conocer cuándo hay una necesidad de información, identificar esta necesidad, saber trabajar con diferentes fuentes y sistemas simbólicos, dominar la sobrecarga de información, evaluarla y discriminar su calidad, organizarla, tener habilidad para la exposición de pensamientos, ser eficaz en el uso de la información para dirigir el problema y saber comunicar a otros la información encontrada. Y estar capacitado para el autoaprendizaje y el trabajo colaborativo (Cabero, 1998). Y todo ello conlleva el uso y dominio de instrumentos tecnológicos para desarrollar su quehacer cotidiano profesional.

En los entornos telemáticos deben diseñarse las actividades digitales de manera que fomenten el uso y manejo básico de los recursos web del actual milenio, principalmente, aquellas relacionadas con el software social, con la web 2.07. De este modo los estudiantes van adquiriendo las competencias tecnológicas en aquellas habilidades necesarias para gestionar y emplear los recursos telemáticos (Internet, herramientas de comunicación sincrónicas y asincrónicas, aplicaciones de tratamiento de texto, imagen y vídeo) tan necesarios para su futuro académico y profesional.

Actividades tales como, participar en una lista de distribución sobre una temática científica, elaboración y desarrollo de un blog, wiki educativo, consultar bases de datos, tesis doctorales, libros y revistas de investigación en línea para profundizar en los contenidos, manejo de slideshare ${ }^{8}$, por ejemplo, para difundir y compartir su presentaciones e informes de sus ideas y experiencias adquiridas, compartir sus enlace en la web, es decir, sus marcadores (bookmarks) en el ámbito mundial (folksonomies), crear, editar y publicar sus libros electrónicos (ebooks). Igualmente, publicar sus fotografías y vídeos de sus experiencias didácticas y crear un repositorio visual de acceso público. Agregarse a canales RSS $^{9}$ para estar actualizados de acontecimientos y eventos de carácter científicos, o como otras posibilidades visitar virtualmente museos, interactuar en simulaciones tridimensionales educativas, consultar lugares geográficos a través de aplicaciones de cartografía por satélite (satellite tools).

\section{CONCLUSIÓN}

A modo de conclusión y de acuerdo con Área (2005), el aprendizaje debe ser un proceso constructivo del conocimiento que el estudiante elabora a través de actividades aprendiendo a resolver situaciones problemáticas en colaboración con otros compañeros. El aprendizaje, en consecuencia, es un proceso de reconstrucción 
de significados que cada individuo realiza en función de su experiencia en una situación dada.

En este sentido, las secuencias de actividades y las e-actividades didácticas presentadas orientadas a la construcción de planes y proyectos de trabajos encaminados a la resolución de problemas en contextos cercanos al estudiante; la construcción del conocimiento a partir de las ideas y experiencias de éstos; la creación de comunidades de conocimientos colaborativos virtuales; el fomento del aprendizaje autónomo; el desarrollo de competencias relacionadas con la búsqueda, el análisis y organización de información procedentes de diversas fuentes y su análisis crítico para la construcción y reelaboración del conocimiento social por parte de los propios estudiantes, la utilización de los recursos telemáticos relacionados con el software, son criterios que se deberían tener en cuenta en la elaboración de instrumentos de análisis didácticos de las estrategias de enseñanza de cursos universitarios en la red Internet.

El uso de las tecnologías Web 2.0 con los nativos e inmigrantes digitales en el ámbito socio-educativo, pueden ayudar y potenciar la constitución y consolidación de equipos de trabajo, facilitar el diálogo e intercambio de ideas, experiencias entre los diferentes grupos sociales, la indagación e investigación educativa, la reflexión colectiva, la interacción y la participación social (López Meneses, 2009).

Las e-actividades como recursos didácticos pueden crear comunidades de conocimiento interculturales y ayudar al estudiante a ser un agente transformador de su entorno social, dinamizador del ciberespacio universitario y generador de redes sociales.

\section{NOTAS}

1 Sánchez Bravo, A. (2001). Internet y la sociedad europea de la información: implicaciones para los ciudadanos. Sevilla: Universidad de Sevilla, p. 42.

2 Bartolomé, A. (1996). Preparando para un nuevo modo de conocer. En EDUTEC. Revista electrónica de Tecnología educativa, $\mathrm{n}^{0}$ 4. Disponible en http://www.uib. es/depart/gte/edutec-e/revelec4/revelec4.html (consulta 2010, 24 de marzo).

3 Varis, T. (1996). "Educar para la sociedad de la información". Telos: Cuadernos de comunicación, Tecnología y Sociedad, 48, p. 79.

4 Gros, B.; García, I.; Lara, P. (2009). El desarrollo de herramientas de apoyo para el trabajo colaborativo en entornos virtuales de aprendizaje. En RIED, vol. $12 \mathrm{n}^{0}$ 2, p. 116. [consulta 2010, 5 de abril).

5 Su dirección web es: http://es.wikipedia.org/wiki/Mapa conceptual 
6 Para más información sobre Web 2.0 se puede consultar:

Web 2.0 Conference 2004: http://www.web2con.com/web2con/

Web 2.0 Conference 2005: http://www.web2con.com/pub/w/40/coverage.html

Web 2.0 Conference 2006: http://www.web2con.com/

7 Fuente: http://internality.com/web2o/

$8 \quad$ Slideshare es un espacio gratuito donde los usuarios pueden enviar presentaciones Powerpoint u OpenOffice, que luego quedan almacenadas en formato Flash para ser visualizadas online. Es una opción interesante para compartir presentaciones en la red. Admite archivos de hasta $20 \mathrm{Mb}$ de peso, sin transiciones entre diapositivas.

9 RSS («Rich Site Summary» o «Really Simple Syndication»). Es un formato basado en el XML de acuerdo a especificaciones de la "Wordd Wide Consortium" que sirve para distribuir titulares de noticias y contenidos. Gracias a RSS, el usuario dispone de los titulares de diferentes de sitios web sin la necesidad de conectarse uno por uno a todos ellos, sabiendo en esta forma en cada momento qué sitios web han actualizado sus contenidos, cuáles no y qué contenidos han sido actualizados (Serrano, Román y Cabero, 2005).

\section{REFERENCIAS BIBLIOGRÁFICAS}

Aebli, H. (1988). 12 formas básicas de enseñar. Madrid: Narcea.

Aguaded, J. I.; López Meneses, E. (2009). La blogosfera educativa: nuevos espacios universitarios de innovación y formación del profesorado en el contexto europeo. Revista electrónica Interuniversitaria de formación del profesorado. REIFOP, 12 (3), (165-172) [en línea] Disponible en: http://www.aufop.com/aufop/revistas/ arta/digital/138/1263 (consulta 2010, 22 de enero).

Área, M. (2005). La educación en el laberinto tecnológico. De la estructura a las máquinas digitales. Barcelona: Ediciones Universitarias de Barcelona. Octaedro.

Ballesteros, C.; López Meneses, E.; Torres, L. $\mathrm{M}^{\mathrm{a}}$. (2004). Las plataformas virtuales: escenarios alternativos para la formación. Edutec '2004. Congreso internacional sobre educación y tecnologías de la información y la comunicación: Educar con tecnologías, de lo excepcional a lo cotidiano. [en línea] Disponible en: http://www.edutec2004.lmi.ub.es/ pdf/195.pdf (consulta 2010, 22 de enero).

Barberá, E. (coord.) (2001). La incógnita de la educación a distancia. ICE de la Universidad de Barcelona. Horsori.

Bartolomé, A. (1996). Preparando para un nuevo modo de conocer. EDUTEC. Revista electrónica de Tecnología educativa, $\mathrm{n}^{\circ} 4$. [en línea] Disponible en: http://www.uib.es/depart/gte/edutece/revelec4/revelec4.html (consulta 2010, 24 de marzo).

Cabero, J. (1998). Usos e integración de los medio audiovisuales y las nuevas tecnologías en el vitae. En: R. Pérez (coord.). Educación y tecnologías de la comunicación (47-67). Oviedo: Servicio de Publicaciones de la Universidad de Oviedo.

Cabero, J. (2003). Principios pedagógicos, psicológicos y sociológicos del trabajo colaborativo: su proyección en la telenseñanza. En: F. Martínez. Redes de comunicación en la enseñanza. 
Las nuevas perspectivas del trabajo corporativo (129-156). Barcelona: Paidós.

Cabero, J. (2006). Comunidades virtuales para el aprendizaje. Su utilización en la enseñanza.EDUTEC,RevistaElectrónica de Tecnología Educativa, 20. [en línea] Disponible en: http://edutec.rediris. es/Revelec2/revelec20/cabero20.htm (consulta 2010, 29 de marzo).

Cabero, J.; Gisbert, M. (Dirs). (2002). Materiales formativos multimedia en la Red. Guía práctica para su diseño. Sevilla: SAV de la Universidad de Sevilla.

Cabero, J.; López Meneses, E.; otros. (2004). ¿Cómo mejorar la práctica profesional de los docentes universitarios? Algunos recursos y utilidades telemáticas. PíxelBit. Revista de Medios y Educación, 22, (5-23).

Cabero, J.; Pérez, A. (2005). Estrategias didácticas para la red: estrategias centradas en la individualización de la enseñanza, estrategias centradas en el trabajo colaborativo, y estrategias para la enseñanza en grupo. [en línea] Disponible en: http://tecnologiaedu. us.es $/ \mathrm{mec} 2005 / \mathrm{html} /$ lista cursos.htm (consulta 2010, 30 de marzo).

Cabero, J.; Valverde. J.; López Meneses, E. (2009). Instrumento didáctico para la valoración de los cursos universitarios en red. En: Roig, R. (Dir.). Investigar desde un contexto educativo innovador (53-71). Alicante: Marfil.

Cañal, P. (2000). Las actividades de enseñanza. Un esquema de clasificación. Investigación en la Escuela, 40, (5-21).

Del Castillo, J.; Barberán, O. (2001). Mapas Conceptuales en Matemáticas. [en línea] Disponible en: http://www.netdidactica. com/articulos/mapas.htm (consulta 2008, 29 de abril).

García Aretio, L. (2006). Nuevos ambientes de aprendizaje. Editorial de BENED. Boletín Electrónico de Noticias de
Educación a Distancia, junio. [en línea] Disponible en: http://www.uned.es/ catedraunesco-ead/bened/benedgo. html (consulta 2010, 24 de marzo).

García Carrasco, J.; García del Dujo, A. (1996). Teoría de la Educación. Salamanca: Universidad de Salamanca.

Gros, B. (2004). La construcción del conocimiento en la red: límites y posibilidades. Teoría de la educación: educación y cultura en la Sociedad de la información, 5. [en línea] Disponible en: http://www3.usal.es/ teoriaeducacion/ (consulta 2010, 29 de marzo).

Gros, B.; García, I.; Lara, P. (2009). El desarrollo de herramientas de apoyo para el trabajo colaborativo en entornos virtuales de aprendizaje. RIED, Revista Iberoamericana de Educación a Distancia, vol. 12, $\mathrm{n}^{\mathrm{0}} 2$, (115-138).

Guiter, M.; Giménez, F. (2000). Trabajo cooperativo en entornos virtuales de aprendizaje. En: Duart, J. (Coord.). Aprender en la virtualidad (113-134). Barcelona: Gedisa.

Imbernón, F. (1994). La formación y el desarrollo profesional del profesorado. Hacia una nueva cultura profesional. Barcelona: Grao.

López Meneses, E. (2009). Guía didáctica para la formación e innovación docente con blogs en el marco europeo. Sevilla: Edición digital @tres.

Novak, J. D. (2000). The Theory Underlying Concept Maps and How to Construct Them. [en línea] Disponible en: http:// cmap.coginst.uwf.edu/info/ (consulta 2008, 29 de abril).

Novak, J. D.; Gowin, D. B. (1999). Aprendiendo a aprender. Barcelona: Ediciones Martínez Roca, S.A.

Román, P. (2003). La flexibilización de los espacios de aprendizaje a través de entornos de trabajo colaborativos telemáticos. III Congreso Internacional Virtual de Educación. [en línea] Disponible en: http://tecnologiaedu. 
us.es/bibliovir/pdf/ROMAN.pdf (consulta 2010, 29 de marzo).

Sánchez Bravo, A. (2001). Internet y la sociedad europea de la información: implicaciones para los ciudadanos. Sevilla: Universidad de Sevilla.

Sevillano García, M. L. (1997). La formación inicial del profesorado en medios de comunicación y nuevas tecnologías. Profesorado: Revista de currículum y formación del profesorado, 1, (49-60).

Torres, L. (2005). Elementos que deben contener las páginas web educativas. Píxel-Bit.Revista de Mediosy Educación, 25. [en línea] Disponible en: http:// www.sav.us.es/pixelbit/articulos/n25/ n25art/art2508.htm (consulta 2005, 10 de enero).
Travé, G; Pozuelos, F.; Cañal, P. (2006). ¿Cómo enseñar investigando? Análisis de las percepciones de tres equipos docentes con diferentes grupos de desarrollo profesional. Revista Iberoamericana de Educación, 39, 5, (1-24).

Valverde, J. (2002). Herramientas de comunicación sincrónica y asincrónica. En: Cabero, J.; Aguaded, J. I. (Dir.). Educar en red Internet como recurso para la educación (57-81). Málaga: Aljibe.

Varis, T. (1996). Educar para la sociedad de la información. Telos: Cuadernos de comunicación, Tecnología y Sociedad, 48, (79).

\section{PERFIL ACADÉMICO Y PROFESIONAL DE LOS AUTORES}

Miguel A. Martín Sánchez. Doctor en Pedagogía por la Universidad de Salamanca con premio extraordinario, Máster por la Universidad Antonio de Nebrija de Madrid y Máster por la Universidad Pablo de Olavide de Sevilla. Actualmente trabaja como profesor en el Departamento de Ciencias de la Educación de la Universidad de Extremadura, en España. Imparte docencia en la Facultad de Formación del Profesorado, en el área de Teoría e Historia de la Educación. Sus contribuciones se centran en la formación del profesorado, la acción educativa, y la reflexión pedagógica de los espacios educativos desde la Teoría de la Educación.

E-mail: miguelmartin@unex.es

Eloy López Meneses. Doctor por la Universidad de Sevilla y profesor en la Universidad Pablo de Olavide de Sevilla. Sus contribuciones se centran en la formación del profesorado y la utilización de las nuevas tecnologías aplicadas a la educación. Es autor de numerosos artículos en revistas especializadas, monografías y capítulos de libros. Asimismo, ha participado en numerosos congresos nacionales e internacionales.

E-mail: eloylope@unex.es 
DIRECCIÓN DE LOS AUTORES:

Dpto. Ciencias de la Educación

Facultad de Formación del Profesorado

Universidad de Extremadura

Avda. Universidad s/n

10071, Cáceres, España

Fecha de recepción del artículo: 07/04/11

Fecha de aceptación del artículo: 25/07/11

\section{Como citar este artículo:}

Martín Sánchez, M. A.; López Meneses, E. (2012). La sociedad de la información y la formación del profesorado. E-actividades y aprendizaje colaborativo. RIED. Revista Iberoamericana de Educación a Distancia, volumen 15, nº 1, pp. 15-35. 